\title{
AWARENESS COMMUNICATIONS BY ENTERTAINING TOY DOLL AGENTS
}

\author{
Kazuyuki Saitoh ${ }^{12}$, Tomoko Yonezawa ${ }^{3}$, and Kenji Mase ${ }^{1}$ \\ 1 ATR Media Information Science Laboratories, \\ 2 Graduate School of Kanazawa Institute of Technology, \\ 3 ATR MIC Research Laboratories (currently with NTT Cyber Space Laboratories) \\ $\{k a z, m a s e\} @ a t r . c o . j p$
}

\begin{abstract}
In this paper, we propose a sensor-doll system that provides multiple users at remote locations with an awareness communication channel. A doll is used as the interface agent of the local user, and this agent is connected to a remote doll by local and/or wide area networks. The doll sends out information on the local ambient activities and the user's intentional interactions to the remote agent and, at the same time, displays the received remote activities by adapting its presentation to the local context.

Musical sound expression is used to display the remote awareness, mixing the local response and remote activities. Music also provides an entertaining and sympathetic intimacy with the doll and eventually the remote user.

The design and implementation of the networked sensor-doll, equipped with various tactile sensors and a PC, are described in detail. We also discuss issues of awareness communication and give preliminary experimental results.
\end{abstract}

Key words: Awareness communication, toy interface agent, sensor doll, musical interaction, and context awareness

\section{Introduction}

People communicate with each other through various expressive media such as letters, figures, speech, gestures, and existence itself. We utilize the different functions of these media appropriately depending on the situation and our purpose. Network and computer technologies have made it possible to communicate with remote persons by telephone, e-mail, videoconference, groupware, and the World Wide Web. Sharing awareness of the existence

The original version of this chapter was revised: The copyright line was incorrect. This has been corrected. The Erratum to this chapter is available at DOI: 10.1007/978-0-387-35660-0_65 
and activities of each other is important for establishing initial and ambient communication channels between remote people. Communication is also enriched by sharing sensuous affective feelings such as excitement, calmness, and intimacy, which can be partially estimated with haptic and biological measures.

This paper proposes a networked toy-shape interface agent system that can support both awareness communication and affective communication. The toy can recognize various contexts and detect ambient activities of users and environmental factors by using visual, audio, tactile and dynamics sensors that work with an installed PC. The recognized contexts and activities are communicated between agents and displayed to each user by means of musical expressions. The anthropomorphic embodiment of the agent as a stuffed toy is chosen to assist the user in interacting with the agent intimately by touching, moving and holding its body. The toy is designed to have only one actuator, an audio speaker, to represent the internal situation, local response and remote user's situation.

One application of this system is to facilitate remote awareness communication, for instance, between grandparents and grandchildren: as the child plays with the toy, it can display the activity to his/her remote grandparents.

In this paper, related works will be described first, and then the system design will be presented. The preliminary experimental results will be briefly described, followed by the conclusions.
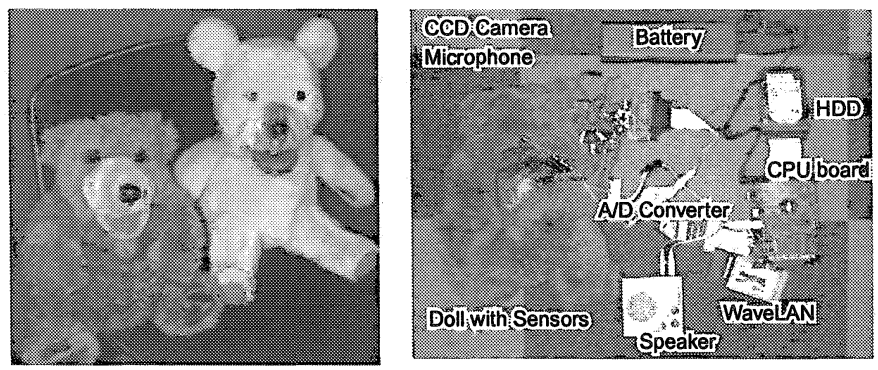

Figure 1. Sensor-dolls and disassembled parts

\section{Related Works}

"in-Touch"[1] is a roller device that connects remote places for tactile communication using physical expressions. It connects remote users through direct manipulation of the roller, a manipulatable haptic device. Our proposal is to use an agent to connect remote users and to display awareness. The Aware-home project [2] has been proposed to develop awareness 
sensitive houses. Affective computing [3] uses biological measures to display emotional information. The current design of our sensor-equipped toy employs tactile sensors such as touch and bend. Biological meters may be used in our toys in the future.

Yonezawa et al. [4] has developed a sensor-doll interface system, named "Com-Music," that has its own internal "mind" states that reflect different contexts, such as sleeping, awareness, chatting, and enjoyment. The user's multimodal interaction with this passive doll is interpreted and responds by musical sound expressions that change depending on the situation's context. We propose extending Com-Music by adding network capability to provide an awareness communication function between remote users.

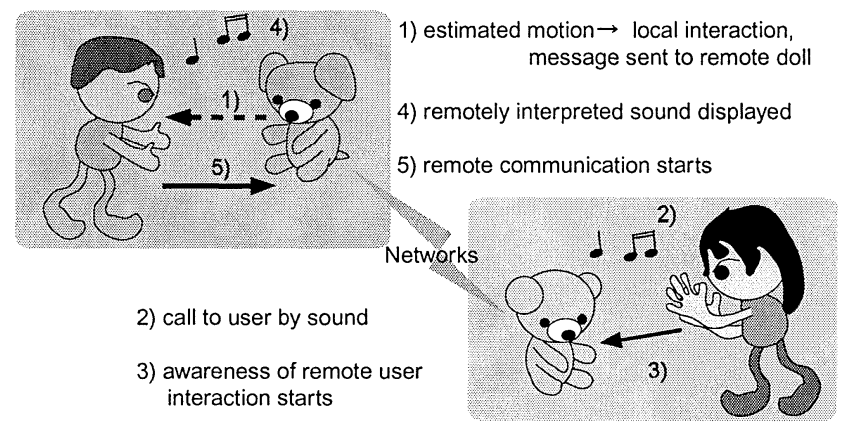

Figure 2. Networked communication using sensor-dolls

\section{Design Issues}

There are several issues in designing an awareness communication agent:

(1) choice of expression,

(2) agent attribution and ownership for user,

(3) influence and use of remote context,

(4) distinction of remote and local interactions in agent expression,

(5) interpretation and mapping of interaction to expression,

(6) hardware and software choice and system construction, and

(7) enjoyment and entertainment.

These considerations are interdependent, and it is not easy to find an optimum solution for general-purpose usage because there are too many possible applications.

For our system, we envision a user group of young people who like music or those who are readily adaptable to music listening in daily life, such as young children. Music is a good medium for adaptation to mobile situations, as is apparent in the use of favorite tunes as the musical ringer on many mobile phones. Music is also often used to provide a specific 
background atmosphere. Music has a special characteristic in that it is subject to multiple interpretations. It allows the listener to take control of the interpretation to some extent, thus providing active immersion in interaction. (issue 1)

We designed the agent as a surrogate of the local user. In other words, the local user is the master of the agent. Such ownership is important in agent design to clarify the policy of information handling. We assume that a personal agent with a toy interface can establish a longer partnership through daily intimate interactions. Once a close partnership is established, it is natural to handle incoming messages by translating and interpreting the local contexts and owner's preferences. (issue 2)

The toy has a basic function of context awareness that changes the internal states as the interaction intensity progresses. We focus on ambient activities for awareness communication, such as room occupation and human movements for purposes other than explicit communication. This corresponds to the earlier internal state or weak intensity stage of the contexts. The mood change of the user can also provide useful awareness information that is not easily transmitted by conventional communication media such as e-mail and voice phone. The toy agents communicate with each other by exchanging the local contexts in terms of internal states and preprocessed interaction data in order to understand the similarities and differences of remote contexts. The remote context's influence in system design will be described later. (issue 3 )

The toy responds and expresses with musical sounds and notes. A multiplicity of interpretation becomes a disadvantage if we want to display the differences between the local and remote interactions. Therefore, we exploit multiple timbres to express them distinctly. (issue 4)

The other three issues, i.e. mapping, system construction, and entertainment, depend heavily on system implementation, which will be described in detail later.

\section{System Design}

The original Com-Music system [4] contains five states of interaction levels (IL) from 0 to 4 (Figure 3), corresponding to sleep, awareness, talking, play, and crazy, respectively. The IL changes as the user intensifies the interaction with the doll. For example, if the user touches the doll for several seconds when it is sleeping, it wakes up and replies with a short voice-like sound segment. 
We have added a few triggers to the finite state transition model to adopt network communication features, as illustrated in Figure 3 by underlines. For example, when the remote interaction level is 2 or 3 , the local interaction level moves from IL0 to IL1. The local doll displays the remote interactions by different timbres and voices, as shown in Table 1, according to the local interaction levels. Thus, at the initial stage, remote users can be aware of a partner's situation and then progress to the joint jam-session level (i.e., IL3 for both) if they wish to play. We have designed the music sequencer so that it adjusts the remote signals in tempo and in harmonic chord to orchestrate plausible resulting music.

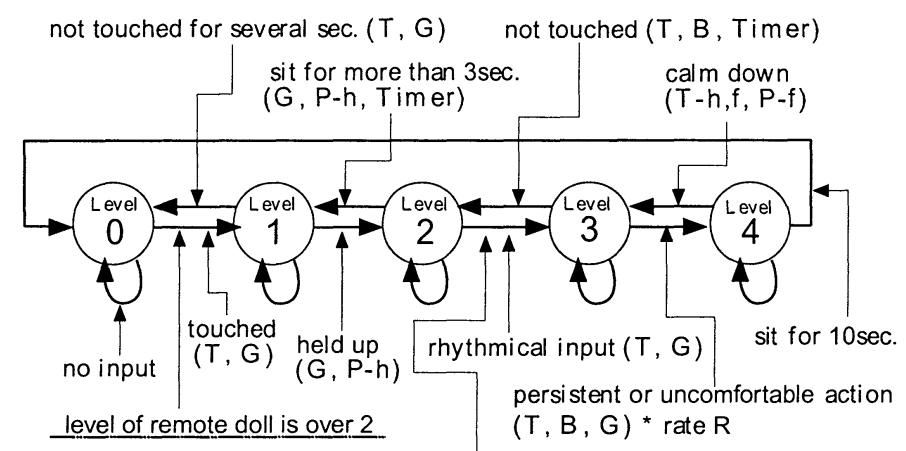

syncronized input against remote sound (B)

$G=G$-force sensor, $T=$ Touch sensor, $P-h=$ Hip proximity sensor, $P-f=$ Face proximity sensor, $B=$ Bend sensor, $T-h=$ Head touch sensor, $T-f=$ Face touch sensor

Figure 3. Transition model of interaction level

\subsection{Implementation Overview}

The Sensor-Doll has an on-board computer with a Celeron $400 \mathrm{MHz}$ CPU and many kinds of sensors, including a USB CCD camera in its face, a USB microphone in its ear, a small USB loud speaker, a G-Force sensor in its belly, proximity sensors on its face and hips, and touch bend sensors in its arms, legs, back, front and head (Figure 4). The sensors are connected through an $\mathrm{A} / \mathrm{D}$ converter (PCMCIA) to the computer. The system is run on Windows 2000 and DirectX 8.0 and was developed in VisualC++6.0 with the DirectMusic toolkit. The local system is connected to the remote system via a wireless LAN using UDP (user diagram protocol). A program diagram is illustrated in Figure 5. As shown in the figure, the dolls exchange their interaction levels and preprocessed sensor readings. The entire process from sensor input to music output can be performed at $25-30 \mathrm{~Hz}$ solely by the PC inside the doll. 


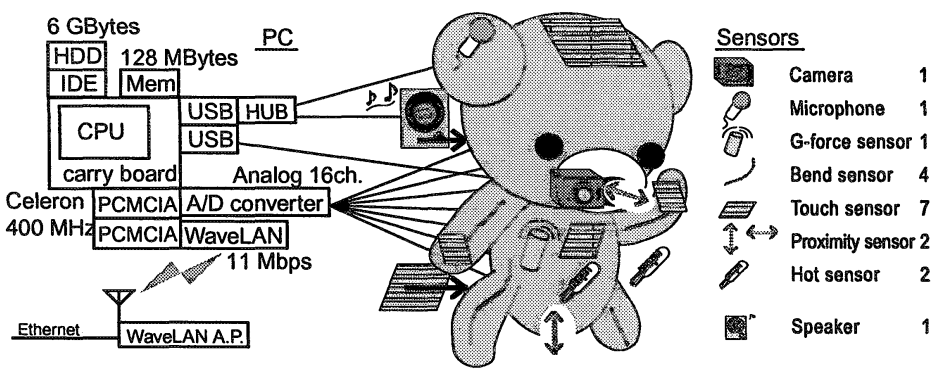

Figure 4. Sensor-doll hardware configuration

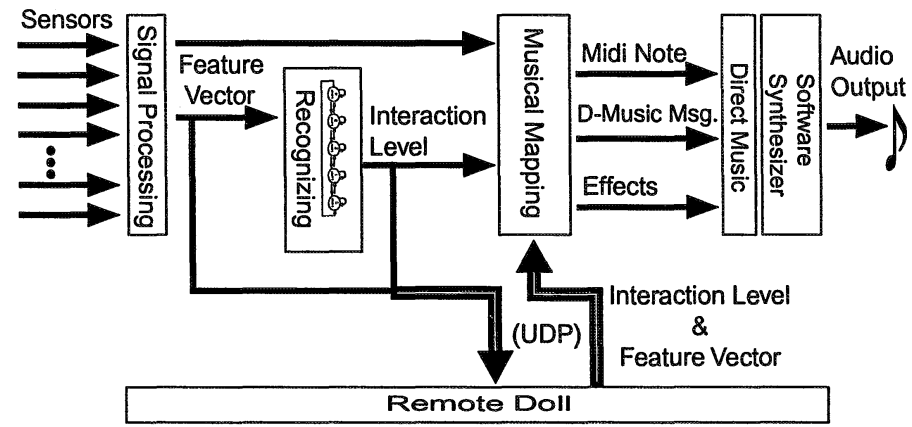

Figure 5. Data processing diagram of sensor-doll

Table 1. Remote and Local Interaction Matrix

\begin{tabular}{|c|c|c|c|c|}
\hline Loca IL & base sand & sand of local interaction & Rernote IL & influence on remote doll \\
\hline \multirow[t]{2}{*}{$\begin{array}{l}\text { 0: Sleeping, and looking } \\
\text { about }\end{array}$} & \multirow[t]{2}{*}{ breath } & \multirow[t]{2}{*}{$\begin{array}{l}\text { Respiratory sound becomes quiet if } \\
\text { moverment of an object is recognized }\end{array}$} & 0 & $\begin{array}{l}\text { Recognition of movement of an object } \\
\text { outputs saund. }\end{array}$ \\
\hline & & & $2 \circ 3$ & IL is set to 1. \\
\hline \multirow[t]{2}{*}{ 1: Wake up and greeting } & & \multirow[t]{2}{*}{$\begin{array}{l}\text { Replies of voice-like saund acoording to } \\
\text { the level of body tauching }\end{array}$} & 2 & $\begin{array}{l}\text { Lowsound is output acoording to the } \\
\text { level of body touding }\end{array}$ \\
\hline & & & 3 & $\begin{array}{l}\text { Sound is output acoording to the level of } \\
\text { body touching. }\end{array}$ \\
\hline \multirow[t]{2}{*}{$\begin{array}{l}\text { 2: Playing inythm } \\
\text { peraussion }\end{array}$} & \multirow[t]{2}{*}{ equalized breath } & \multirow[t]{2}{*}{$\begin{array}{l}\text { Sands of dfferent inythmperaussion } \\
\text { at specific places of the body. }\end{array}$} & 2 & $\begin{array}{l}\text { Sounds of dfferent inythm peraussion at } \\
\text { specific places of the body. }\end{array}$ \\
\hline & & & 3 & $\begin{array}{l}\text { Sands of different shat music phrases } \\
\text { at specific places of the bodx. }\end{array}$ \\
\hline \multirow[t]{2}{*}{ 3. Jamsession } & \multirow[t]{2}{*}{ main malodys } & \multirow[t]{2}{*}{$\begin{array}{l}\text { Sands of different short music phrases } \\
\text { at specific places of the body. }\end{array}$} & 2 & $\begin{array}{l}\text { The main malody is influenoed by the } \\
\text { frequency of body touching. }\end{array}$ \\
\hline & & & 3 & $\begin{array}{l}\text { Sounds of different short music phrases } \\
\text { at specific places of the body. }\end{array}$ \\
\hline 4: Untrace, uncontrollable & inharmonic tone & & & \\
\hline
\end{tabular}




\section{Preliminary Experiments}

We conducted a preliminary experiment with a female adult subject in her 20's who was not aware of the system's design. The experiment consisted of three test stages: a free local play test without any instructions or explanations of the system to observe the naive responses; a free local play test after a system design explanation of the five internal states; and a remote interaction test without telling the subject the doll is connected to another system and a remote user in a different room.

The subject used the system freely, holding and touching various parts of the doll in the first stage. She was able to move to IL2 without any particular problem. In the second test stage, the subject was able to control movement around all of the states. In the final test, she recognized that there was a sound response by someone else and replied with action and sound by touching the doll. The remote interaction is shown in Figure 6. Some synchronized response can be observed in the figure. At first, the subject plays her own timing, but when she suddenly becomes aware that the sound is made by the experimenter's interaction, she synchronizes the play to make the response appear to be her own.

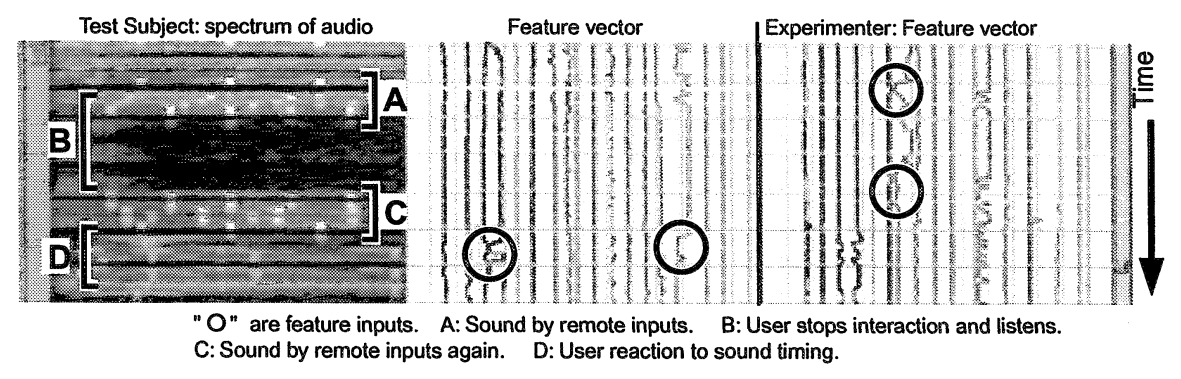

Figure 6. Remote interaction example

\section{Conclusions}

We have proposed an awareness communication system with musical enjoyment using interactive toy agents. We developed a sensor doll system that is equipped with a computer and various sensors such as a camera, microphone, accelerometer, and touch-sensitive sensors. The doll recognizes its own situation and the activities of a local user as well as a remote situation and a remote user's activities by receiving the state and activity signals from the remote agent. More experimentation is necessary to confirm the usefulness of the doll agent for remote existence awareness and context 
awareness communication. So far, we have observed that users enjoy the toy shape and music display through tactile interaction.

In the future, we would like to increase the number of internal states so that the doll is able to express a greater variety of music depending on the context. For example, Clarkson et al. [5] proposed a method of location awareness using a camera, microphone, and G-force sensor with a Hidden Markov Model. The use of more complex contexts such as user's identity, location, and time will be useful for providing intimate remote awareness.

This paper described an application of the sensor-doll agent in musical remote awareness communication, focusing on its musical and tactile communication capability. However, its basic function of getting the interaction information to a closer position to the user can still be used in a greater variety of applications. For instance, it can be integrated for context recognition in a ubiquitous sensor environment, where user's activities are recorded for personal memory, experience sharing with others, and further assistance for actions by the intelligent environment.

\section{Acknowledgements}

We thank Norihiro Hagita, director, and the members of the ATR Media Information Science Laboratories for their support of and discussions on this research. We also thank the Kanazawa Institute of Technology and Prof. Hattori for their invaluable support of the student internship program that allowed Kazuyuki Saitoh to perform this research at ATR. This research was supported in part by the Telecommunications Advancement Organization of Japan.

\section{References}

[1] S.Brave, H.Ishii, A Dahley,"Tangible Interfaces for Remote Communication and Collaboration", Proceedings of CSCW '98, pp. 169-178, ACM,1998.

[2] K.Nagel, C.D.Kidd, T. O'Connell, A.Dey, G.D.Abowd, "The Family Intercom: Developing a Context-Aware Audio Communication System", Proceedings of Ubicomp2001, pp. 176-183, 2001.

[3] R.W.Picard, "Affective Computing", The MIT Press,1998.

[4] T.Yonezawa, B.Clarkson, M.Yasumura, K. Mase, "Context-aware Sensor-Doll as a Music Expression Device", Extended Abstracts of CHI2001, pp. 307-308, 2001

[5] B. Clarkson, K.Mase, A. Pentland, "Recognizing User Context via Wearable Sensors, Proceedings of ISWC2000, pp. 565-569, 2000. 